umented. Based on the results of this study, deer are not as large a reservoir for $E$ coli $\mathrm{O} 157: \mathrm{H} 7$ as cattle, but they likely play an important role in its spread, especially where deer live near cattle.

(Appl Environ Microbiol. 2001;67:1218-1224) J. R. Fischer, T. Zhao, M. P. Doyle, et al.

\section{AVIATION, SPACE, AND ENVIRONMENTAL MEDICINE}

\section{Effect of daily versus intermittent exposure on heat acclimation}

Anyone who has ever participated in an outdoor activity on a hot humid day understands the benefits of heat acclimation. Many studies that define the physiological changes that take place during this process have been reported. There is less literature, however, describing the best method with which to become heat acclimated. The authors of this study test the hypothesis that daily exposure to heat is more efficacious for heat acclimation than intermittent exposure.

Fourteen healthy athletes (mean age 23.5 years) were randomized into 2 groups of 7 subjects. Each group contained 5 females and 2 males, and there were no significant differences between the groups. Before the sessions started, the maximum exercise capacity of each subject was determined by measuring peak oxygen consumption, $\mathrm{VO}_{2}$, while exercising on a rowing ergometer. Each subject then exercised on the rowing ergometer at a power output equal to $70 \%$ of their maximum ability. One group exercised for 30 minutes per day for 10 consecutive days. The other group exercised for 30 minutes per day every Monday, Wednesday, and Friday for a total of 10 sessions. All sessions took place in an environmental chamber set at $38^{\circ} \mathrm{C}$ and $70 \%$ relative humidity. Rectal temperature, skin temperature, heart rate, ratings of perceived exertion, and sweat rate were all recorded.

Results show consecutive exposure to be superior to intermittent exposure in nearly every category. Rectal temperature decreased significantly in both groups by day 5 , but fell to $96 \%$ of its predicted plateau value in the consecutive exposure group and to only $26 \%$ of the predicted plateau in the intermittent exposure group. Heart rate decreased to $63 \%$ of the predicted plateau in the consecutive exposure group compared to only $16 \%$ in the intermittent exposure group. Skin temperature was also significantly lower in the continuous exposure group. Ratings of perceived exertion and sweat rate were not significantly different between the groups.

The main limitation of this study is the small sample size. Otherwise, the methods are clear and consistent, and the statistical analysis seems sound. In addition, the authors of this article present a very thorough review of relevant literature, which adds to the validity of the design and the results. Despite the study's small size, the results of this study agree with the results of previous similar studies. Larger studies should be done to confirm these results.

(Aviat Space Environ Med. 2000;71:385-390) N. Gill, B. Phed, and G. Sleivert.

\section{CLINICAL INFECTIOUS DISEASES}

\section{Risk factors for severe pulmonary and disseminated coccidioidomycosis: Kern County, California, 1995-1996}

In most cases, patients with coccidioidomycosis (CM) suffer nothing more than mild, influenza-like symptoms, but in severe cases, this disease can be fatal. As with many diseases, knowing who is at risk to develop severe complications can help direct management decisions. It is well known that the San Joaquin Valley in California is hyperendemic for CM, and much of our data about $\mathrm{CM}$ comes from research done in this area. This article provides a recent update of CM in Kern County, which is located within the San Joaquin Valley.

The authors conducted both a 2-year population-based surveillance program for $\mathrm{CM}$ and a retrospective case-control study of patients with CM. The surveillance program identified all the individuals between January 1, 1995, and December 31, 1996, in Kern County who were older than 18 years and had culture, histopathologic, molecular, or serological evidence of Coccidioides immitis, the fungus that causes CM. From this data set, patients with positive diagnoses between January 1, 1995, and September 30, 1996, were classified as having mild CM, severe pulmonary CM, or disseminated CM. Mild CM was defined as a mild flulike illness. These patients served as case controls. For someone to be classified as having severe pulmonary CM, there had to be radiographic evidence of pneumonia that resulted in hospitalization. Patients classified as having disseminated CM were those with extrapulmonary or miliary CM. Enrolled patients were then contacted by telephone and asked to complete a standard questionnaire. Data on demographic characteristics, outdoor activities, dust exposure, past medical history, tobacco and alcohol use, occupation, socioeconomic status, and antifungal treatment for $\mathrm{CM}$ were obtained. Also recorded was the number of days missed from work or school as a result of having CM.

The surveillance program identified 905 persons newly diagnosed with CM. Of these, 682 met inclusion criteria for the case-control study, and 380 were enrolled. Both univariate and multivariate analyses were performed. Univariate analysis revealed several risk factors for severe pulmonary $\mathrm{CM}$ and disseminated CM. Patients who acquired severe pulmonary CM were more likely to be older, more likely to be agricultural workers, and more likely to have a longer disease course. Risk factors for disseminated CM were being male, being black, being pregnant, or having a longer disease course.

Multivariate analyses showed slightly different results. Risk factors for severe pulmonary $\mathrm{CM}$, as defined by an odds ratio 